\title{
Iterative methods for variational inequality problems and fixed point problems of a countable family of strict pseudo-contractions in a $q$-uniformly smooth Banach space
}

\author{
Pongsakorn Sunthrayuth ${ }^{1,2}$ and Poom Kumam ${ }^{1,2^{*}}$
}

* Correspondence: poom. kum@kmutt.ac.th

'Department of Mathematics, Faculty of Science, King Mongkut's University of Technology Thonburi (KMUTT) Bangmod, Bangkok 10140, Thailand

Full list of author information is available at the end of the article

\begin{abstract}
In this article, we introduce iterative methods (implicit and explicit) for finding a common fixed point set of a countable family of strict pseudo-contractions, which is a unique solution of some variational inequality. Furthermore, we prove the strong convergence theorems of such iterative scheme in a q-uniformly smooth Banach space which admits a weakly sequentially continuous generalized duality mapping. The results presented in this article extend and generalize the corresponding results announced by Yamada and Ceng et al. from Hilbert spaces to Banach spaces.

Mathematics Subject Classification 2010: 47H09; 47J05; 47J20; 65J15.

Keywords: strict pseudo-contractions, variational inequality, strong convergence theorem, $q$-uniformly smooth Banach space, common fixed point
\end{abstract}

\section{Introduction}

Let $X$ be a real Banach space, and $X^{*}$ be its dual space. Let $U=\{x \in X:\|x\|=1\}$. A Banach space $X$ is said to be strictly convex if $\frac{\|x+y\|}{2}<1$ for all $x, y \in X$ with $\|x\|=\|y\|=$ 1 and $x \neq y$. A Banach space $X$ is called uniformly convex if for each $\varepsilon>0$ there is a $\delta>0$ such that for $x, y \in X$ with $\|x\|,\|y\| \leq 1$ and $\|x-y\| \geq \varepsilon,\|x+y\| \leq 2(1-\delta)$ holds. The $\bmod$ ulus of convexity of $X$ defined by

$$
\delta_{X}(\varepsilon)=\inf \left\{1-\left\|\frac{1}{2}(x+y)\right\|:\|x\|,\|y\| \leq 1,\|x-y\| \geq \varepsilon\right\},
$$

for all $\varepsilon \in[0,2] . X$ is uniformly convex if $\delta_{x}(0)=0$ and $\delta_{x}(\varepsilon)>0$ for all $0<\varepsilon \leq 2$. It is know that every uniformly convex Banach space is strictly convex and reflexive (see [1]). The norm of $X$ is said to be Gâteaux differentiable if the limit

$$
\lim _{t \rightarrow 0} \frac{\|x+t y\|-\|x\|}{t}
$$

exists for each $x, y \in U$. In this case $X$ is smooth. Let $\rho_{X}:[0, \infty) \rightarrow[0, \infty)$ be the modulus of smoothness of $X$ defined by

(C) 2012 Sunthrayuth and Kumam; licensee Springer. This is an Open Access article distributed under the terms of the Creative Commons Attribution License (http://creativecommons.org/licenses/by/2.0), which permits unrestricted use, distribution, and reproduction in any medium, provided the original work is properly cited. 


$$
\rho_{X}(\tau)=\sup \left\{\frac{1}{2}(\|x+y\|+\|x-y\|)-1: x \in U,\|y\| \leq \tau\right\} .
$$

A Banach space $X$ is said to be uniformly smooth if $\frac{\rho_{X}(t)}{t} \rightarrow 0$ as $t \rightarrow 0$. Suppose that $q>1$, then $X$ is said to be $q$-uniformly smooth if there exists $c>0$ such that $\rho_{X}(t) \leq$ $c t^{q}$. It is easy to see that if $X$ is $q$-uniformly smooth, then $q \leq 2$ and $X$ is uniformly smooth. For $q>1$, the generalized duality mapping $J_{q}: X \rightarrow 2^{X^{*}}$ is defined by

$$
J_{q}(x)=\left\{f \in X^{*}:\langle x, f\rangle=\|x\|^{q},\|f\|=\|x\|^{q-1}\right\},
$$

where $\langle\cdot, \cdot\rangle$ denotes the duality pairing between $X$ and $X^{*}$. In particular, $J_{q}=J_{2}$ is called the normalized duality mapping and $J_{q}(x)=\|x\|^{q-2} J_{2}(x)$ for $x \neq 0$. If $X:=H$ is a real Hilbert space, then $J=I$ where $I$ is the identity mapping. Further, we have the following properties of the generalized duality mapping $J_{q}$ :

(1) $J_{q}(x)=\|x\|^{q-2} J_{2}(x)$ for all $x \in X$ with $x \neq 0$.

(2) $J(t x)=t^{q-1} J_{q}(x)$ for all $x \in X$ and $t \in[0, \infty)$.

(3) $J_{q}(-x)=-J_{q}(x)$ for all $x \in X$.

It is well known that if $X$ is smooth, then $J_{q}$ is single-valued, which is denoted by $j_{q}$ (see [1]). The duality mapping $J_{q}$ from a smooth Banach space $X$ into $X^{*}$ is said to be weakly sequentially continuous generalized duality mapping if for all $\left\{x_{n}\right\} \subset X$ with $x_{n} \rightarrow x$ implies $J_{q}\left(x_{n}\right) \rightarrow * J_{q}(x)$.

Let $C$ be a nonempty, closed and convex subset of $X$ and $T$ be a self-mapping of $C$. We denote the fixed points set of the mapping $T$ by $\operatorname{Fix}(T)=\{x \in C: T x=x\}$ and denote $\rightarrow$ and $\rightarrow$ by strong and weak convergence, respectively.

Definition 1.1. A mapping $T: C \rightarrow C$ is said to be:

(i) $\lambda$-strictly pseudocontractive [2], if for all $x, y \in C$ there exists $\lambda>0$ and $j_{q}(x-y)$ $\in J_{q}(x-y)$ such that

$$
\left\langle T x-T y, j_{q}(x-y)\right\rangle \leq\|x-y\|^{q}-\lambda\|(I-T) x-(I-T) y\|^{q},
$$

or equivalently

$$
\left\langle(I-T) x-(I-T) y, j_{q}(x-y)\right\rangle \geq \lambda\|(I-T) x-(I-T) y\|^{q} .
$$

(ii) L-Lipschitzian if for all $x, y \in C$, there exists a constant $L>0$ such that

$$
\|T x-T y\| \leq L\|x-y\| .
$$

If $0<L<1$, then $T$ is a contraction and if $L=1$, then $T$ is a nonexpansive mapping. By the definition, we know that every $\lambda$-strictly pseudocontractive mapping is $\left(\frac{1+\lambda}{\lambda}\right)$-Lipschitzian (see [3]). 
Remark 1.2. Let $C$ be a nonempty subset of a real Hilbert space $H$ and $T: C \rightarrow C$ be a mapping. Then $T$ is said to be $k$-strictly pseudocontractive [2], if for all $x, y \in C$, there exists $k \in[0,1)$ such that

$$
\|T x-T y\|^{2} \leq\|x-y\|^{2}+k\|(I-T) x-(I-T) y\|^{2} .
$$

It is well known that (1.1) is equivalent to the following:

$$
\langle T x-T y, x-y\rangle \leq\|x-y\|-\frac{1-k}{2}\|(I-T) x-(I-T) y\|^{2} .
$$

Let $C$ be a nonempty, closed and convex subset of $X$ and $\Psi: C \rightarrow X$ be a nonlinear mapping. The variational inequality problem is to find $u \in C$ such that

$$
\left\langle\Psi u, j_{q}(v-u)\right\rangle \geq 0, \quad \forall v \in C,
$$

where $j_{q}(v-u) \in J_{q}(v-u)$. The set of solution of variational inequality problem is denoted by $V I(C, \Psi)$. If $X:=H$ is a real Hilbert space, the variational inequality problem reduces to find $u \in C$ such that

$$
\langle\Psi u, v-u\rangle \geq 0, \quad \forall v \in C .
$$

Applications of variational inequalities span as diverse disciplines as differential equations, time-optimal control, optimization, mathematical programming, mechanics, finance and so on (see, e.g., [4,5] for more details). Note that most of the variational problems, including minimization or maximization of functions, variational inequality problems, quasivariational inequality problems, decision and management sciences, and engineering sciences problems can be unified into form (1.2) and (1.3). For more details, we recommend the reader [6-11]. On the author hand, we note that iterative approximation of fixed points of nonexpansive mappings (and of common fixed points of nonexpansive semigroups) have recently been applied to image recovery and signal processing (see, e.g., [12-17]).

A mapping $F: C \rightarrow X$ is said to be accretive if for all $x, y \in C$ there exists $j_{q}(x-y) \in$ $J_{q}(x-y)$ such that

$$
\left\langle F x-F y, j_{q}(x-y)\right\rangle \geq 0 .
$$

For some $\eta>0, F: C \rightarrow X$ is said to be strongly accretive if for all $x, y \in C$ there exists $j_{q}(x-y) \in J_{q}(x-y)$ such that

$$
\left\langle F x-F y, j_{q}(x-y)\right\rangle \geq \eta\|x-y\|^{q} .
$$

Remark 1.3. If $X:=H$ is a real Hilbert space, accretive and strongly accretive mappings coincide with monotone and strongly monotone mappings, respectively.

Let $A$ be a strongly positive bounded linear operator on $H$, that is, there exists a constant $\bar{\gamma}>0$ such that

$$
\langle A x, x\rangle \geq \bar{\gamma}\|x\|^{2}, \quad \text { for all } x \in H .
$$

Remark 1.4. From the definition of operator $A$, we note that a strongly positive bounded linear operator $A$ is a $\|A\|$-Lipschitzian and $\eta$-strongly monotone operator. 
A typical problem is to minimize a quadratic function over the set of the fixed points of a nonexpansive mapping on a real Hilbert space $H$ :

$$
\min _{x \in C} \frac{1}{2}\langle A x, x\rangle-\langle x, u\rangle,
$$

where $C$ is the fixed point set of a nonexpansive mapping $T$ on $H$ and $u$ is a given point in $H$.

In 2006, Marino and Xu [18] introduced and considered the following general iterative method:

$$
x_{n+1}=\alpha_{n} \gamma f\left(x_{n}\right)+\left(I-\alpha_{n} A\right) T x_{n}, \quad \forall n \geq 0,
$$

where $A$ is a strongly positive bounded linear operator on a real Hilbert space $H$. They, proved that, if the sequence $\left\{\alpha_{n}\right\}$ of parameters satisfies appropriate conditions, then the sequence $\left\{x_{n}\right\}$ generated by (1.6) converges strongly to the unique solution of the variational inequality

$$
\left\langle(\gamma f-A) x^{*}, x-x^{*}\right\rangle \leq 0, \quad \forall x \in \operatorname{Fix}(T),
$$

which is the optimality condition for the minimization problem

$$
\min _{x \in C} \frac{1}{2}\langle A x, x\rangle-h(x)
$$

where $C$ is the fixed point set of a nonexpansive mapping $T$ and $h$ is a potential function for $\gamma f$ (i.e., $h^{\prime}(x)=\gamma f(x)$ for all $x \in H$ ).

On the other hand, Yamada [19] introduced a hybrid steepest descent method for a non-expansive mapping $T$ as follows:

$$
x_{n+1}=T x_{n}-\mu \lambda_{n} F\left(T x_{n}\right), \quad \forall n \geq 0,
$$

where $F$ is a $\kappa$-Lipschitzian and $\eta$-strongly monotone operator with constants $\kappa, \eta>$ 0 and $0<\mu<\frac{2 \eta}{\kappa^{2}}$. He proved that if $\left\{\lambda_{n}\right\}$ satisfying appropriate conditions, then the sequence $\left\{x_{n}\right\}$ generated by (1.9) converges strongly to the unique solution of variational inequality

$$
\left\langle F x^{*}, x-x^{*}\right\rangle \geq 0, \quad \forall x \in \operatorname{Fix}(T) .
$$

In 2010, Tian [20] combined the iterative method (1.6) with the Yamada's method (1.9) and considered a general iterative method for a nonexpansive mapping $T$ as follows:

$$
x_{n+1}=\alpha_{n} \gamma f\left(x_{n}\right)+\left(I-\alpha_{n} \mu F\right) T x_{n}, \quad \forall n \geq 0 .
$$

Then he proved that the sequence $\left\{x_{n}\right\}$ generated by (1.11) converges strongly to the unique solution of variational inequality

$$
\left\langle(\gamma f-\mu F) x^{*}, x-x^{*}\right\rangle \leq 0, \quad \forall x \in \operatorname{Fix}(T) .
$$

Very recently, Ceng et al. [21] introduced implicit and explicit iterative schemes for finding the fixed points of a nonexpansive mapping $T$ on a nonempty, closed and convex subset $C$ in a real Hilbert space $H$ as follows: 


$$
x_{t}=P_{C}\left[t \gamma V x_{t}+(I-t \mu F) T x_{t}\right]
$$

and

$$
x_{n+1}=P_{C}\left[\alpha_{n} \gamma V x_{n}+\left(I-\alpha_{n} \mu F\right) T x_{n}\right], \quad \forall n \geq 0,
$$

where $V$ is an $L$-Lipschitzian mapping with a constant $L \geq 0$ and $F$ is a $\kappa$-Lipschitzian and $\eta$-strongly monotone operator with constants $\kappa, \eta>0$ and $0<\mu<\frac{2 \eta}{\kappa^{2}}$. Then they proved that the sequences generated by (1.13) and (1.14) converge strongly to the unique solution of variational inequality

$$
\left\langle(\mu F-\gamma V) x^{*}, x^{*}-x\right\rangle \geq 0, \quad \forall x \in \operatorname{Fix}(T) .
$$

The following questions naturally arise in connection with above results:

Question 1.5. Can Theorem of Ceng et al. [21] be extend from a real Hilbert space to a general Banach space? such as q-uniformly smooth Banach space.

Question 1.6. Can we extend the iterative method of scheme (1.14) to a general iterative scheme define over the set of fixed points of a countable family of strict pseudocontractions.

The purpose of this article is to give the affirmative answers to these questions mentioned above, motivated by Yamada [19], Tian [20] and Ceng et al. [21], we introduce a general iterative method for finding a common fixed point set of a countable family of strict pseudo-contractions, which is a unique solution of some variational inequality. Furthermore, we prove the strong convergence theorems of such iterative scheme in a $q$-uniformly smooth Banach space which admits a weakly sequentially continuous generalized duality mapping. The results presented in this article extend and generalize the corresponding results announced by Yamada [19] and Ceng et al. [21] and many others to Banach spaces.

\section{Preliminaries}

Let $D$ be a nonempty subset of $C$. A mapping $Q: C \rightarrow D$ is said to be sunny if

$$
Q(Q x+t(x-Q x))=Q x
$$

whenever $Q x+t(x-Q x) \in C$ for $x \in C$ and $t \geq 0$. A mapping $Q: C \rightarrow D$ is said to be retraction if $Q x=x$ for all $x \in D$. Furthermore, $Q$ is a sunny nonexpansive retraction from $C$ onto $D$ if $Q$ is a retraction from $C$ onto $D$ which is also sunny and nonexpansive. A retraction $Q$ is said to be orthogonal if for each $x, x-Q x$ is normal to $D$ in the sense of James (see [22]). A subset $D$ of $C$ is called a sunny nonexpansive retraction of $C$ if there exists a sunny nonexpansive retraction from $C$ onto $D$. It is well known that if $X:=H$ is a real Hilbert space, then a sunny nonexpansive retraction $Q_{c}$ is coincident with the metric projection from $X$ onto $C$.

The following lemma concerns the sunny nonexpansive retraction.

Lemma 2.1. [23] Let $C$ be a closed and convex subset of a real q-uniformly smooth Banach space $X$. Let $Q: X \rightarrow C$ be a retraction. Then, $Q$ is an orthogonal retraction if and only if

$$
\left\langle x-Q x, j_{q}(y-Q x)\right\rangle \leq 0, \quad \forall x \in X \text { and } y \in C .
$$


Lemma 2.2. [24] Let $X$ be a real q-uniformly smooth Banach space. Then the following inequality holds:

$$
\|x+y\|^{q} \leq\|x\|^{q}+q\left\langle y, J_{q}(x)\right\rangle+C_{q}\|y\|^{q},
$$

for all $x, y \in X$ and for some $C_{q}>0$.

Lemma 2.3. [25] Suppose that $q>1$. Then the following inequality holds:

$$
a b \leq \frac{1}{q} a^{q}+\left(\frac{q-1}{q}\right) b^{\frac{q}{q-1}}
$$

for arbitrary positive real numbers $a, b$.

Lemma 2.4. [26] Let $\left\{s_{n}\right\}$ be a sequence of nonnegative real numbers, $\left\{a_{n}\right\}$ be $a$ sequence of $[0,1]$ with $\sum_{n=1}^{\infty} a_{n}=\infty,\left\{c_{n}\right\}$ be a sequence of nonnegative real number with $\sum_{n=1}^{\infty} c_{n}<\infty$ and $\left\{b_{n}\right\}$ be a sequence of real numbers with $\lim \sup _{n \rightarrow \infty} b_{n} \leq 0$. Suppose that

$$
s_{n+1}=\left(1-a_{n}\right) s_{n}+a_{n} b_{n}+c_{n}
$$

for all $n \in \mathbb{N}$. Then, $\lim _{n \rightarrow \infty} s_{n}=0$.

Definition 2.5. Let $\left\{T_{n}\right\}$ be a family of mappings from a subset $C$ of a Banach space $X$ into itself with $\bigcap_{n=1}^{\infty} F\left(T_{n}\right) \neq \emptyset$. We say that $\left\{T_{n}\right\}$ satisfies the $A K T T$-condition (see [26]) if for each bounded subset $B$ of $C$,

$$
\sum_{n=1}^{\infty} \sup _{\omega \in B}\left\|T_{n+1} \omega-T_{n} \omega\right\|<\infty .
$$

Lemma 2.6. [26]Suppose that $\left\{T_{n}\right\}$ satisfy the AKTT-condition such that

(i) For each $x \in C,\left\{T_{n}\right\}$ is converge strongly to some point in $C$.

(ii) Let the mapping T:C $\rightarrow C$ defined by $T x=\lim _{n \rightarrow \infty}, T_{n} x$ for all $x \in C$.

Then $\lim _{n \rightarrow \infty} \sup _{\omega \in B}\left\|T \omega-T_{n} \omega\right\|=0$ for each bounded subset $B$ of $C$.

Lemma 2.7. $[27,28]$ Let $C$ be a closed and convex subset of a smooth Banach space $X$. Suppose that $\left\{T_{n}\right\}_{n=1}^{\infty}: C \rightarrow X$ is a family of $\lambda$-strictly pseudocontractive mappings with $\left\{\mu_{m}\right\}_{n=1}^{\infty}$ and $\left\{\mu_{m}\right\}_{n=1}^{\infty}$ is a real sequence in $(0,1)$ such that $\sum_{n=1}^{\infty} \mu_{n}=1$. Then the following conclusions hold:

(i) A mapping G: $C \rightarrow X$ defined by $G:=\sum_{n=1}^{\infty} \mu_{n} T_{n}$ is a $\lambda$-strictly pseudocontractive mapping.

(ii) $\operatorname{Fix}(G)=\bigcap_{n=1}^{\infty} \operatorname{Fix}\left(T_{n}\right)$.

Lemma 2.8. [28] Let $C$ be a closed and convex subset of a smooth Banach space $X$. Suppose that $\left\{S_{k}\right\}_{k=1}^{\infty}: C \rightarrow C$ is a countable family of $\lambda$-strictly pseudocontractive mappings with $\bigcap_{k=1}^{\infty} \operatorname{Fix}\left(S_{k}\right) \neq \emptyset$. For all $n \in \mathbb{N}$, define $T_{n}: C \rightarrow C$ by 
$\left\{\mu_{n}^{k}\right\}$ for all $x \in C$, where $\left\{\mu_{n}^{k}\right\}$ is a family of nonnegative numbers satisfying the following conditions:

(i) $\sum_{k=1}^{n} \mu_{n}^{k}=1$ for all $n \in \mathbb{N}$;

(ii) $\mu^{k}:=\lim _{n \rightarrow \infty} \mu_{n}^{k}>0$ for all $k \in \mathbb{N}$;

(iii) $\sum_{n=1}^{\infty} \sum_{k=1}^{n}\left|\mu_{n+1}^{k}-\mu_{n}^{k}\right|<\infty$.

Then the following hold:

(1) Each $T_{n}$ is a $\lambda$-strictly pseudocontractive mapping.

(2) $\left\{T_{n}\right\}$ satisfies the AKTT-condition.

(3) If $T: C \rightarrow C$ is defined by $T x=\sum_{k=1}^{\infty} \mu^{k} S_{k} x$ for all $x \in C$, then $T x=\lim _{n \rightarrow \infty} T_{n} x$ and $\operatorname{Fix}(T)=\bigcap_{n=1}^{\infty} \operatorname{Fix}\left(T_{n}\right)=\bigcap_{k=1}^{\infty} \operatorname{Fix}\left(S_{k}\right)$.

\section{Main results}

In order to prove our main result, the following lemmas are needed.

Lemma 3.1. Let $C$ be a nonempty, closed and convex subset of a real q-uniformly smooth Banach space X. Let $F: C \rightarrow X$ be a $\kappa$-Lipschitzian and $\eta$-strongly accretive operator with constants $\kappa, \eta>0$. Let $0<\mu<\left(\frac{q \eta}{C_{q} \kappa^{q}}\right)^{\frac{1}{q-1} \text { and }}$ $\tau=\mu\left(\eta-\frac{C_{q} \mu^{q-1} \kappa^{q}}{q}\right)$. Then for $t \in\left(0, \min \left\{1, \frac{1}{\tau}\right\}\right)$, the mapping $S: C \rightarrow X$ define by $S:=(I-t \mu F)$ is a contraction with constant $1-t \tau$.

Proof. Since $0<\mu<\left(\frac{q \eta}{C_{q^{q}}}\right)^{\frac{1}{q-1}}$ with $q>1$ and $t \in\left(0, \min \left\{1, \frac{1}{\tau}\right\}\right)$. This implies that $1-t \tau \in(0,1)$. From Lemma 2.2, for all $x, y \in C$, we have

$$
\begin{aligned}
\|S x-S y\|^{q} & =\|(I-t \mu F) x-(I-t \mu F) y\|^{q} \\
& =\|(x-y)-t \mu(F x-F y)\|^{q} \\
& \leq\|x-y\|^{q}-q t \mu\left\langle F x-F y_{j} j_{q}(x-y)\right\rangle+C_{q} t^{q} \mu^{q}\|F x-F y\|^{q} \\
& \leq\|x-y\|^{q}-q t \mu \eta\|x-y\|^{q}+C_{q} t^{q} \mu^{q} \kappa^{q}\|x-y\|^{q} \\
& \leq\left[1-t \mu\left(q \eta-C_{q} \mu^{q-1} \kappa^{q}\right)\right]\|x-y\|^{q} \\
& =\left[1-t \mu q\left(\eta-\frac{C_{q} \mu^{q-1} \kappa^{q}}{q}\right)\right]\|x-\gamma\|^{q} \\
& \leq\left[1-t \mu\left(\eta-\frac{C_{q} \mu^{q-1} k^{q}}{q}\right)\right]^{q}\|x-\gamma\|^{q} \\
& =(1-t \tau)^{q}\|x-\gamma\|^{q} .
\end{aligned}
$$

It follows that

$$
\|S x-S y\| \leq(1-t \tau)\|x-y\| .
$$

Hence, we have $S:=(I-t \mu F)$ is a contraction with a constant $1-t \tau$. 
Lemma 3.2. Let $C$ be a nonempty, closed and convex subset of a real q-uniformly smooth Banach space $X$ which admits weakly sequentially continuous generalized duality mapping $j_{q}$ from $X$ into $X^{*}$. Let $T: C \rightarrow C$ be a nonexpansive mapping. Then, for all $\left\{x_{n}\right\} \subset C$, if $x_{n}-x$ and $x_{n}-T x_{n} \rightarrow 0$, then $x=T x$.

Proof. From Lemma 2.2, for all $x \in C$, we have

$$
\begin{aligned}
\left\|x-T x-\left(x_{n}-T x_{n}\right)\right\|^{q} & =\left\|\left(x_{n}-x\right)+\left(T x-T x_{n}\right)\right\|^{q} \\
& \leq\left\|x_{n}-x\right\|^{q}+q\left\langle T x-T x_{n}, j_{q}\left(x_{n}-x\right)\right\rangle+C_{q}\left\|T x-T x_{n}\right\|^{q} \\
& \leq\left\langle x_{n}-x, j_{q}\left(x_{n}-x\right)\right\rangle+q\left\langle T x-T x_{n}, j_{q}\left(x_{n}-x\right)\right\rangle+C_{q}\left\langle x-x_{n}, j_{q}\left(x-x_{n}\right)\right\rangle .
\end{aligned}
$$

Taking the limit as $n \rightarrow \infty$ in both sides and noting that $j_{q}$ is weakly sequentially continuous generalized duality mapping. Then, $\|x-T x\|^{q} \leq 0$, this implies that $x=T x$.

\subsection{Implicit iteration scheme}

Let $C$ be a nonempty, closed and convex subset of a real $q$-uniformly smooth Banach space $X$. Let $Q_{C}$ be a sunny nonexpansive retraction from $X$ onto $C$. Let $F: C \rightarrow X$ be a $\kappa$-Lipschitzian and $\eta$-strongly accretive operator with constants $\kappa, \eta>0, V: C \rightarrow X$ be an $L$-Lipschitzian mapping with a constant $L \geq 0$ and $T: C \rightarrow C$ be a nonexpansive mapping such that $\operatorname{Fix}(T) \neq \varnothing$. Let $0<\mu<\left(\frac{q \eta}{C_{q} \kappa^{q}}\right)^{\frac{1}{q-1}}$ and $0 \leq \gamma L<\tau$, where $\tau=\mu\left(\eta-\frac{C_{q} \mu^{q-1} \kappa^{q}}{q}\right)$. For each $t \in\left(0, \min \left\{1, \frac{1}{\tau}\right\}\right)$, we define the mapping $S_{t}: C$ $\rightarrow C$ by

$$
S_{t} x:=Q_{C}[t \gamma V x+(I-t \mu F) T x], \quad \forall x \in C .
$$

It is easy to see that $S_{t}$ is a contraction. Indeed, from Lemma 3.1, for all $x, y \in C$, we have

$$
\begin{aligned}
\left\|S_{t} x-S_{t} y\right\| & =\| Q_{C}[t \gamma V x+(I-t \mu F) T x]-Q_{C}[t \gamma V y+(I-t \mu F) T y] \\
& \leq\|[t \gamma V x+(I-t \mu F) T x]-[t \gamma V y+(I-t \mu F) T y]\| \\
& \leq t \gamma\|V x-V y\|+\|(I-t \mu F)(T x-T \gamma)\| \\
& \leq t \gamma L\|x-\gamma\|+(1-t \tau)\|x-\gamma\| \\
& =(1-(\tau-\gamma L) t)\|x-\gamma\| .
\end{aligned}
$$

Hence, $S_{t}$ has a unique fixed point, denoted by $x_{t}$, which uniquely solve the fixed point equation

$$
x_{t}=Q_{C}\left[t \gamma V x_{t}+(I-t \mu F) T x_{t}\right]
$$

The following proposition summarizes the properties of the net $\left\{x_{t}\right\}$.

Proposition 3.3. Let $C$ be a nonempty, closed and convex subset of a real q-uniformly smooth Banach space $X$. Let $Q_{c}$ be a sunny nonexpansive retraction from $X$ onto $C$. Let $F: C \rightarrow X$ be a $\kappa$-Lipschitzian and $\eta$-strongly accretive operator with constants $\kappa, \eta>$ $0, V: C \rightarrow X$ be an L-Lipschitzian mapping with a constant $L \geq 0$ and $T: C \rightarrow C$ be a nonexpansive mapping such that $\operatorname{Fix}(T) \neq \varnothing$. Let $0<\mu<\left(\frac{q \eta}{C_{q} \kappa^{q}}\right)^{\frac{1}{q-1}}$ and $0 \leq \gamma L$ 
$<\tau$, where $\tau=\mu\left(\eta-\frac{C_{q} \mu^{q-1} \kappa^{q}}{q}\right)$.

then

(i) $\left\{x_{t}\right\}$ is bounded for each $t \in\left(0, \min \left\{1, \frac{1}{\tau}\right\}\right)$.

(ii) $\lim _{t \rightarrow 0}\left\|x_{t}-T x_{t}\right\|=0$.

(iii) $\left\{x_{t}\right\}$ defines a continuous curve from $\left(0, \min \left\{1, \frac{1}{\tau}\right\}\right)$ into $C$.

\section{Proof.}

(i) Taking $\bar{x} \in \operatorname{Fix}(T)$. Then, we have

$$
\begin{aligned}
\left\|x_{t}-\bar{x}\right\| & =\left\|Q_{C}\left[t \gamma V x_{t}+(I-t \mu F) T x_{t}\right]-Q_{C} \bar{x}\right\| \\
& \leq\left\|\left[t \gamma V x_{t}+(I-t \mu F) T x_{t}\right]-\bar{x}\right\| \\
& =\left\|t\left(\gamma V x_{t}-\mu F \bar{x}\right)+(I-t \mu F)\left(T x_{t}-\bar{x}\right)\right\| \\
& \leq t\left\|\gamma V x_{t}-\mu F \bar{x}\right\|+(1-t \tau)\left\|T x_{t}-\bar{x}\right\| \\
& \leq t \gamma\left\|V x_{t}-V \bar{x}\right\|+t\|\gamma V \bar{x}-\mu F \bar{x}\|+(1-t \tau)\left\|x_{t}-\bar{x}\right\| \\
& \leq(1-(\tau-\gamma L) t)\left\|x_{t}-\bar{x}\right\|+t\|\gamma V \bar{x}-\mu F \bar{x}\| .
\end{aligned}
$$

It follows that

$$
\left\|x_{t}-\bar{x}\right\| \leq \frac{\|\gamma V \bar{x}-\mu F \bar{x}\|}{\tau-\gamma L} .
$$

Hence, $\left\{x_{t}\right\}$ is bounded, so are $\left\{V x_{t}\right\}$ and $\left\{F T x_{t}\right\}$.

(ii) By definition of $\left\{x_{t}\right\}$, we have

$$
\begin{aligned}
\left\|x_{t}-T x_{t}\right\| & =\left\|Q_{C}\left[t \gamma V x_{t}+(I-t \mu F) T x_{t}\right]-Q_{C} T x_{t}\right\| \\
& \leq\left\|t \gamma V x_{t}+(I-t \mu F) T x_{t}-T x_{t}\right\| \\
& =t\left\|\gamma V x_{t}-\mu F T x_{t}\right\| \rightarrow 0 \quad \text { as } \quad t \rightarrow 0 .
\end{aligned}
$$

(iii) Take $t, t_{0} \in\left(0, \min \left[1, \frac{1}{\tau}\right]\right)$. From Lemma 3.1, we have

$$
\begin{aligned}
\left\|x_{t}-x_{t_{0}}\right\| & =\left\|Q_{C}\left[t \gamma V x_{t}+(I-t \mu F) T x_{t}\right]-Q_{C}\left[t_{0} \gamma V x_{t_{0}}+(I-t \mu F) T x_{t_{0}}\right]\right\| \\
& \leq\left\|\left[t \gamma V x_{t}+(I-t \mu F) T x_{t}\right]-\left[t_{0} \gamma V x_{t_{0}}+(I-t \mu F) T x_{t_{0}}\right]\right\| \\
& =\left\|\left(t-t_{0}\right) \gamma V x_{t}+t_{0} \gamma\left(V x_{t}-V x_{t_{0}}\right)-\left(t-t_{0}\right) \mu F T x_{t}+\left(I-t_{0} \mu F\right)\left(T x_{t}-T x_{t_{0}}\right)\right\| \\
& \leq\left(\gamma\left\|V x_{t}\right\|+\mu\left\|F T x_{t}\right\|\right)\left|t-t_{0}\right|+\left(1-(\tau-\gamma L) t_{0}\right)\left\|x_{t}-x_{t_{0}}\right\| .
\end{aligned}
$$

It follows that

$$
\left\|x_{t}-x_{t_{0}}\right\| \leq \frac{\left(\gamma\left\|V x_{t}\right\|+\mu\left\|F T x_{t}\right\|\right)}{(\tau-\gamma L) t_{0}}\left|t-t_{0}\right|
$$


Since $\left\{V x_{t}\right\}$ and $\left\{F T x_{t}\right\}$ is bounded. Hence, $\left\{x_{t}\right\}$ defines a continuous curve from $\left(0, \min \left\{1, \frac{1}{\tau}\right\}\right)$ into $C$.

Theorem 3.4. Let $C$ be a nonempty, closed and convex subset of a real q-uniformly smooth Banach space $X$ which admits a weakly sequentially continuous generalized duality mapping $j_{q}$ from $X$ into $X^{*}$. Let $Q_{c}$ be a sunny nonexpansive retraction such that $Q_{c}$ is an orthogonal from $X$ onto $C$. Let $F: C \rightarrow X$ be a $\kappa$-Lipschitzian and $\eta$ strongly accretive operator with constants $\kappa, \eta>0, V: C \rightarrow X$ be an L-Lipschitzian mapping with constant $L \geq 0$ and $T: C \rightarrow C$ be a nonexpansive mapping such that Fix

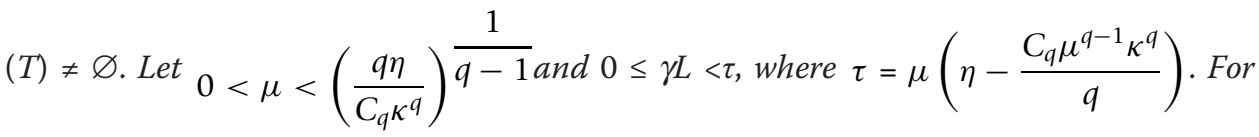
each $t \in\left(0, \min \left\{1, \frac{1}{\tau}\right\}\right)$, let $\left\{x_{t}\right\}$ defined by (3.1), then $\left\{x_{t}\right\}$ converges strongly to $x^{* *} \in$ $\operatorname{Fix}(T)$ as $t \rightarrow 0$, which $x^{*}$ is the unique solution of the variational inequality

$$
\left\langle(\mu F-\gamma V) x^{*}, j_{q}\left(x^{*}-z\right)\right\rangle \leq 0, \quad \forall z \in \operatorname{Fix}(T) .
$$

Proof. We observe that

$$
\begin{aligned}
\frac{C_{q} \mu^{q-1} \kappa^{q}}{q}>0 & \Leftrightarrow \eta-\frac{C_{q} \mu^{q-1} \kappa^{q}}{q}<\eta \\
& \Leftrightarrow \mu\left(\eta-\frac{C_{q} \mu^{q-1} \kappa^{q}}{q}\right)<\mu \eta \\
& \Leftrightarrow \tau<\mu \eta .
\end{aligned}
$$

It follows that

$$
0 \leq \gamma L<\tau<\mu \eta .
$$

First, we show the uniqueness of solution of the variational inequality (3.3). Suppose that $\tilde{x}, x^{*} \in \operatorname{Fix}(T)$ are solutions of (3.3), then

$$
\left\langle(\mu F-\gamma V) x^{*}, j_{q}\left(x^{*}-\tilde{x}\right)\right\rangle \leq 0
$$

and

$$
\left\langle(\mu F-\gamma V) \tilde{x}, j_{q}\left(\tilde{x}-x^{*}\right)\right\rangle \leq 0 .
$$

Adding up (3.5) and (3.6), we have

$$
\begin{aligned}
0 & \geq\left\langle(\mu F-\gamma V) x^{*}-(\mu F-\gamma V) \tilde{x}, j_{q}\left(x^{*}-\tilde{x}\right)\right\rangle \\
& =\mu\left\langle F x^{*}-F \tilde{x}, j_{q}\left(x^{*}-\tilde{x}\right)\right\rangle-\gamma\left\langle V x^{*}-V \tilde{x}, j_{q}\left(x^{*}-\tilde{x}\right)\right\rangle \\
& \geq \mu \eta\left\|x^{*}-\tilde{x}\right\|^{q}-\gamma\left\|V x^{*}-V \tilde{x}\right\|\left\|x^{*}-\tilde{x}\right\|^{q-1} \\
& \geq(\mu \eta-\gamma L)\left\|x^{*}-\tilde{x}\right\|^{q} .
\end{aligned}
$$

Note that (3.4) implies that $x^{*}=\tilde{x}$ and the uniqueness is proves. Below, we use $x^{*}$ to denote the unique solution of the variational inequality (3.3).

Next, we show that $x_{t} \rightarrow x^{*}$ as $t \rightarrow 0$. Setting $y_{t}=t \gamma V x_{t}+(I-t \mu F) T x_{t}$, where $t \in\left(0, \min \left\{1, \frac{1}{\tau}\right\}\right)$. Then, we can rewrite (3.1) as $x_{t}=Q C y t$. Assume that $\left\{t_{n}\right\} \subset$ 
$(0,1)$ is a sequence such that $t_{n} \rightarrow 0$ as $n \rightarrow \infty$. Putting $x_{n}:=x_{t_{n}}$ and $y_{n}: y_{t_{n}}$. For $z \in$ $\operatorname{Fix}(T)$, we note that

$$
\begin{aligned}
x_{n}-z & =Q_{C} y_{n}-y_{n}+y_{n}-z \\
& =Q_{C} y_{n}-y_{n}+t_{n}\left(\gamma V x_{n}-\mu F z\right)+\left(I-t_{n} \mu F\right)\left(T x_{n}-z\right) .
\end{aligned}
$$

By Lemma 2.1, we have

$$
\left\langle Q_{C} y_{n}-y_{n}, j_{q}\left(Q_{C} y_{n}-z\right)\right\rangle \leq 0
$$

It follows from (3.7) and (3.8) that

$$
\begin{aligned}
\left\|x_{n}-z\right\|^{q} & =\left\langle Q_{C} y_{n}-y_{n}, j_{q}\left(Q_{C} y_{n}-z\right)\right\rangle+\left\langle\left(I-t_{n} \mu F\right)\left(T x_{n}-z\right), j_{q}\left(x_{n}-z\right)\right\rangle \\
& +t_{n}\left\langle\gamma V x_{n}-\mu F z, j_{q}\left(x_{n}-z\right)\right\rangle \\
& \leq\left(1-t_{n} \tau\right)\left\|x_{n}-z\right\|^{q}+t_{n}\left\langle\gamma V x_{n}-\mu F z_{,} j_{q}\left(x_{n}-z\right)\right\rangle .
\end{aligned}
$$

Thus, we have

$$
\begin{aligned}
\left\|x_{n}-z\right\|^{q} & \leq \frac{1}{\tau}\left\langle\gamma V x_{n}-\mu F z, j_{q}\left(x_{n}-z\right)\right\rangle \\
& =\frac{1}{\tau}\left\{\gamma\left\langle V x_{n}-V z, j_{q}\left(x_{n}-z\right)\right\rangle+\left\langle\gamma V z-\mu F z, j_{q}\left(x_{n}-z\right)\right\rangle\right\} \\
& \leq \frac{1}{\tau}\left\{\gamma L\left\|x_{n}-z\right\|^{q}+\left\langle\gamma V z-\mu F z, j_{q}\left(x_{n}-z\right)\right\rangle\right\}
\end{aligned}
$$

which implies that

$$
\left\|x_{n}-z\right\|^{q} \leq \frac{1}{\tau-\gamma L}\left\langle\gamma V z-\mu F z, j_{q}\left(x_{n}-z\right)\right\rangle .
$$

In particular, we have

$$
\left\|x_{n_{i}}-z\right\|^{q} \leq \frac{1}{\tau-\gamma L}\left\langle\gamma V z-\mu F z, j_{q}\left(x_{n_{i}}-z\right)\right\rangle .
$$

By reflexivity of a Banach space $X$ and boundedness of $\left\{x_{n}\right\}$, there exists a subsequence $\left\{x_{n_{i}}\right\}$ of $\left\{x_{n}\right\}$ such that $x_{n_{i}} \rightarrow \tilde{x}$ as $i \rightarrow \infty$. Since Banach space $X$ has a weakly sequentially continuous generalized duality mapping and by (3.9), we obtain $x_{n_{i}} \rightarrow \tilde{x}$. By Proposition 3.3 (ii), we have $x_{n}-T x_{n} \rightarrow 0$ as $n \rightarrow \infty$. Hence, it follows from Lemma 3.2 that $\tilde{x} \in \operatorname{Fix}(T)$.

Next, we show that $\tilde{x}$ solves the variational inequality (3.3). We note that

$$
x_{t}=Q_{C} y_{t}=Q_{C} y_{t}-y_{t}+t \gamma V x_{t}+(I-t \mu F) T x_{t}
$$

we derive that

$$
(\mu F-\gamma V) x_{t}=\frac{1}{t}\left(Q_{C} y_{t}-y_{t}\right)-\frac{1}{t}(I-T) x_{t}+\mu\left(F x_{t}-F T x_{t}\right) .
$$

Since $I-T$ is accretive (i.e., $\left\langle(I-T) x-(I-T) y, j_{q}(x-y)\right\rangle \geq 0$, for $\left.x, y \in C\right)$. For all $z \in$ $\operatorname{Fix}(T)$, it follows from (3.10) and Lemma 2.1 that

$$
\begin{aligned}
\left\langle(\mu F-\gamma V) x_{t} j_{q}\left(x_{t}-z\right)\right\rangle= & \frac{1}{t}\left\langle Q_{C} y_{t}-y_{t}, j_{q}\left(Q_{C} y_{t}-z\right)\right\rangle-\frac{1}{t}\left\langle(I-T) x_{t}-(I-T) z_{,} j_{q}\left(x_{t}-z\right)\right\rangle \\
& +\mu\left\langle F x_{t}-F T x_{t}, j_{q}\left(x_{t}-z\right)\right\rangle \\
\leq & \mu\left\langle F x_{t}-F T x_{t}, j_{q}\left(x_{t}-z\right)\right\rangle \\
\leq & \mu\left\|F x_{t}-F T x_{t}\right\|\left\|x_{t}-z\right\|^{q-1} \\
\leq & \left\|x_{t}-T x_{t}\right\| M
\end{aligned}
$$


where $M>0$ is a constant such that $M=\sup \left\{\mu \kappa\left\|x_{t}-z\right\|^{q-1}\right\}$, where $t \in\left(0, \min \left\{1, \frac{1}{\tau}\right\}\right)$.

Now, replacing $t$ in (3.11) with $t_{n}$ and taking the limit as $n \rightarrow \infty$, we noticing that $x_{t_{n}}-T x_{t_{n}} \rightarrow \tilde{x}-T \tilde{x}=0$ for $\tilde{x} \in \operatorname{Fix}(T)$, we obtain $\left\langle(\mu F-\gamma V) \tilde{x}, j_{q}(\tilde{x}-z)\right\rangle \leq 0$. That is $\tilde{x} \in \operatorname{Fix}(T)$ is the solution of variational inequality (3.3). Consequently, $\tilde{x}=x^{*}$ by uniqueness. Therefore $x_{t} \rightarrow x^{*}$ as $t \rightarrow 0$. This completes the proof.

\subsection{Explicit iteration scheme}

Theorem 3.5. Let $C$ be a nonempty, closed and convex subset of a real q-uniformly smooth Banach space $X$ which admits a weakly sequentially continuous generalized duality mapping $j_{q}$ from $X$ into $X$ *. Let $Q_{c}$ be a sunny nonexpansive retraction such that $Q_{c}$ is an orthogonal from $X$ onto $C$. Let $F: C \rightarrow X$ be a $\kappa$-Lipschitzian and $\eta$-strongly accretive operator with constants $\kappa, \eta>0, V: C \rightarrow X$ be an L-Lipschitzian mapping with constant $L \geq 0$. Let $0<\mu<\left(\frac{q \eta}{C_{q} \kappa^{q}}\right)^{\frac{1}{q-1}}$ and $0 \leq \gamma L<\tau$, where $\tau=\mu\left(\eta-\frac{C_{q} \mu^{q-1} \kappa^{q}}{q}\right)$. Let $\left\{T_{n}\right\}_{n=1}^{\infty}: C \rightarrow C$ be a family of $\lambda$-strict pseudo-contractions with $0<\lambda<1$. Define a mapping $S_{n} x:=\left(1-\gamma_{n}\right) x+\gamma_{n} T_{n} x$ for all $x \in C$ and $n \geq 1$. Assume that $\Omega:=\bigcap_{n=1}^{\infty} \operatorname{Fix}\left(T_{n}\right) \neq \emptyset$. Let $\left\{x_{n}\right\}$ be a sequence defined by $x_{1} \in C$ and

$$
x_{n+1}=Q_{C}\left[\alpha_{n} \gamma V x_{n}+\left(I-\alpha_{n} \mu F\right) S_{n} x_{n}\right], \quad \forall n \geq 1,
$$

where $\left\{\alpha_{n}\right\}$ and $\left\{\gamma_{n}\right\}$ are sequences in $(0,1)$ which satisfy the following conditions:

(C1) $\lim _{n \rightarrow \infty} \alpha_{n}=0$ and $\sum_{n=1}^{\infty} \alpha_{n}=\infty$;

(C2) either $\sum_{n=1}^{\infty}\left|\alpha_{n+1}-\alpha_{n}\right|<\infty$ or $\lim _{n \rightarrow \infty} \frac{\alpha_{n+1}}{\alpha_{n}}=1$;

(C3) $0<\gamma_{n} \leq \delta, \delta=\min \left\{1,\left(\frac{q \lambda}{C_{q}}\right)^{\frac{1}{q-1}}\right\}$ and $\sum_{n=1}^{\infty}\left|\gamma_{n+1}-\gamma_{n}\right|<\infty$.

Suppose in addition that $\left\{T_{n}\right\}_{n=1}^{\infty}$ satisfies the AKTT-condition. Let T: $C \rightarrow C$ be the mapping defined by $T x=\lim _{n \rightarrow \infty} T_{n} x$ for all $x \in C$ and suppose that $\operatorname{Fix}(T)=\bigcap_{n=1}^{\infty} \operatorname{Fix}\left(T_{n}\right)$. Then the sequence $\left\{x_{n}\right\}$ defined by (3.12) converges strongly to $x^{*}$ $\in \operatorname{Fix}(T)$ as $n \rightarrow \infty$, which $x^{*}$ is the unique solution of the variational inequality

$$
\left\langle(\mu F-\gamma V) x^{*}, j_{q}\left(x^{*}-z\right)\right\rangle \leq 0, \quad \forall z \in \operatorname{Fix}(T) .
$$

Proof. From the condition $(C 1)$, we may assume, without loss of generality, that $\alpha_{n} \leq \min \left\{1, \frac{1}{\tau}\right\}$ for all $n \in \mathbb{N}$. First, we show that $\left\{x_{n}\right\}$ is bounded. From Lemma 2.2 and the condition (C3), for all $x, y \in C$, we have

$$
\begin{aligned}
& \left\|S_{n} x-S_{n} y\right\|^{q}=\left\|\left(1-\gamma_{n}\right) x+\gamma_{n} T_{n} x-\left[\left(1-\gamma_{n}\right) y+\gamma_{n} T_{n} y\right]\right\|^{q} \\
& =\left\|x-y-\gamma_{n}\left[x-y-\left(T_{n} x-T_{n} y\right)\right]\right\|^{q} \\
& \leq\|x-y\|^{q}-q \gamma_{n}\left\langle x-y-\left(T_{n} x-T_{n} y\right), j_{q}(x-y)\right\rangle+C_{q} \gamma_{n}^{q}\left\|x-y-\left(T_{n} x-T_{n} y\right)\right\|^{q} \\
& =\|x-\gamma\|^{q}-q \gamma_{n}\|x-\gamma\|^{q}+q \gamma_{n}\left\langle T_{n} x-T_{n} \gamma_{,} j_{q}(x-\gamma)\right\rangle+C_{q} \gamma_{n}^{q}\left\|x-\gamma-\left(T_{n} x-T_{n} \gamma\right)\right\|^{q} \\
& \leq\|x-\gamma\|^{q}+q \gamma_{n}\left(\|x-\gamma\|^{q}-\lambda\left\|x-\gamma-\left(T_{n} x-T_{n} \gamma\right)\right\|^{q}\right)-q \gamma_{n}\|x-\gamma\|^{q} \\
& +C_{q} \gamma_{n}^{q}\left\|x-y-\left(T_{n} x-T_{n} y\right)\right\|^{q} \\
& =\|x-\gamma\|^{q}+\left(C_{q} \gamma_{n}^{q}-q \gamma_{n} \lambda\right)\left\|x-\gamma-\left(T_{n} x-T_{n} y\right)\right\|^{q} \\
& \leq\|x-y\|^{2} \text {. }
\end{aligned}
$$


It follows that $\left\|S_{n} x-S_{n} y\right\| \leq\|x-y\|$, which implies that $S_{n}$ is nonexpansive and Fix $\left(T_{n}\right)=\operatorname{Fix}\left(S_{n}\right)$. Taking $\bar{x} \in \Omega$. Then we have

$$
\begin{aligned}
\left\|x_{n+1}-\bar{x}\right\| & =\left\|Q_{C}\left[\alpha_{n} \gamma V x_{n}+\left(I-\alpha_{n} \mu F\right) S_{n} x_{n} x_{n}\right]-Q_{C} \bar{x}\right\| \\
& \leq\left\|\alpha_{n} \gamma V x_{n}+\left(I-\alpha_{n} \mu F\right) S_{n} x_{n} x_{n}-\bar{x}\right\| \\
& =\left\|\alpha_{n}\left(\gamma V x_{n}-\mu F \bar{x}\right)+\left(I-\alpha_{n} \mu F\right)\left(S_{n} x_{n}-\bar{x}\right)\right\| \\
& \leq \alpha_{n}\left\|\gamma V x_{n}-\mu F \bar{x}\right\|+\left(1-\alpha_{n} \tau\right)\left\|S_{n} x_{n}-\bar{x}\right\| \\
& \leq \alpha_{n} \gamma\left\|V x_{n}-V \bar{x}\right\|+\alpha_{n}\|\gamma V \bar{x}-\mu F \bar{x}\|+\left(1-\alpha_{n} \tau\right)\left\|x_{n}-\bar{x}\right\| \\
& \leq\left(1-(\tau-\gamma L) \alpha_{n}\right)\left\|x_{n}-\bar{x}\right\|+\alpha_{n}\|\gamma V \bar{x}-\mu F \bar{x}\| \\
& =\left(1-(\tau-\gamma L) \alpha_{n}\right)\left\|x_{n}-\bar{x}\right\|+(\tau-\gamma L) \alpha_{n} \frac{\|\gamma V \bar{x}-\mu F \bar{x}\|}{\tau-\gamma L} .
\end{aligned}
$$

By induction, we have $\left\|x_{n}-\bar{x}\right\| \leq \max \left\{\left\|x_{1}-\bar{x}\right\|, \frac{\|\gamma V \bar{x}-\mu F \bar{x}\|}{\tau-\gamma L}\right\}, \quad \forall n \geq 1$.

Hence, $\left\{x_{n}\right\}$ is bounded, so are $\left\{V x_{n}\right\}$ and $\left\{F S_{n} x_{n}\right\}$.

Next, we show that $\left\|x_{n+1}-x_{n}\right\| \rightarrow 0$ as $n \rightarrow \infty$. Since

$$
\begin{aligned}
\left\|S_{n+1} x_{n+1}-S_{n} x_{n}\right\| & \leq\left\|S_{n+1} x_{n+1}-S_{n+} x_{n}\right\|+\left\|S_{n+1} x_{n}-S_{n} x_{n}\right\| \\
& \leq\left\|x_{n+1}-x_{n}\right\|+\left\|\left(1-\gamma_{n+1}\right) x_{n}+\gamma_{n+1} T_{n+1} x_{n}-\left[\left(1-\gamma_{n}\right) x_{n}+\gamma_{n} T_{n} x_{n}\right]\right\| \\
& =\left\|x_{n+1}-x_{n}\right\|+\left\|\left(\gamma_{n+1}-\gamma_{n}\right)\left(T_{n+1} x_{n}-x_{n}\right)+\gamma_{n}\left(T_{n+1} x_{n}-T_{n} x_{n}\right)\right\| \\
& \leq\left\|x_{n+1}-x_{n}\right\|+\left|\gamma_{n+1}-\gamma_{n}\right|\left\|T_{n+1} x_{n}-x_{n}\right\|+\gamma_{n}\left\|T_{n+1} x_{n}-T_{n} x_{n}\right\| .
\end{aligned}
$$

On the other hand, we have

$$
\begin{aligned}
\left\|x_{n+2}-x_{n+1}\right\|= & \left\|Q_{C}\left[\alpha_{n+1} \gamma V x_{n+1}+\left(I-\alpha_{n+1} \mu F\right) S_{n+1} x_{n+1}\right]-Q_{C}\left[\alpha_{n} \gamma V x_{n}+\left(I-\alpha_{n} \mu F\right) S_{n} x_{n}\right]\right\| \\
\leq & \left\|\left[\alpha_{n+1} \gamma V x_{n+1}+\left(I-\alpha_{n+1} \mu F\right) S_{n+1} x_{n+1}\right]-\left[\alpha_{n} \gamma V x_{n}+\left(I-\alpha_{n} \mu F\right) S_{n} x_{n}\right]\right\| \\
= & \| \alpha_{n+1} \gamma\left(V x_{n+1}-V x_{n}\right)+\left(\alpha_{n+1}-\alpha_{n}\right) \gamma V x_{n}+\left(I-\alpha_{n+1} \mu F\right)\left(S_{n+1} x_{n+1}-S_{n} x_{n}\right) \\
& +\left(\alpha_{n}-\alpha_{n+1}\right) \mu F S_{n} x_{n} \| \\
\leq & \alpha_{n+1} \gamma L\left\|x_{n+1}-x_{n}\right\|+\left|\alpha_{n+1}-\alpha_{n}\right|\left(\gamma\left\|V x_{n}\right\|+\mu\left\|F S_{n} x_{n}\right\|\right) \\
& +\left(1-\alpha_{n+1} \tau\right)\left\|S_{n+1} x_{n+1}-S_{n} x_{n}\right\| .
\end{aligned}
$$

Substituting (3.14) into (3.15), we obtain

$$
\begin{aligned}
\left\|x_{n+2}-x_{n+1}\right\| \leq & \alpha_{n+1} \gamma L\left\|x_{n+1}-x_{n}\right\|+\left|\alpha_{n+1}-\alpha_{n}\right|\left(\gamma\left\|V x_{n}\right\|+\mu\left\|F S_{n} x_{n}\right\|\right) \\
& +\left(1-\alpha_{n+1} \tau\right)\left[\left\|x_{n+1}-x_{n}\right\|+\left|\gamma_{n+1}-\gamma_{n}\right|\left\|T_{n+1} x_{n}-x_{n}\right\|+\gamma_{n}\left\|T_{n+1} x_{n}-T_{n} x_{n}\right\|\right] \\
\leq & \left(1-(\tau-\gamma L) \alpha_{n+1}\right)\left\|x_{n+1}-x_{n}\right\|+\left|\alpha_{n+1}-\alpha_{n}\right| M_{1}+\left|\gamma_{n+1}-\gamma_{n}\right| M_{2} \\
& +\left\|T_{n+1} x_{n}-T_{n} x_{n}\right\|,
\end{aligned}
$$

where $M_{1}=\sup _{n \geq 1}\left\{\gamma\left\|V x_{n}\right\|, \mu\left\|F S_{n} x_{n}\right\|\right\}$ and $M_{2}=\sup _{n \geq 1}\left\{\left\|T_{n+1} x_{n}-x_{n}\right\|\right\}$. It follows from the conditions $(C 2),(C 3)$ and Lemma 2.4 that

$$
\lim _{n \rightarrow \infty}\left\|x_{n+1}-x_{n}\right\|=0
$$

Next, we show that $\left\|x_{n}-S x_{n}\right\| \rightarrow 0$ as $n \rightarrow \infty$. For any bounded subset $B$ of $C$, we observe that

$$
\begin{aligned}
\sup _{\omega \in B}\left\|S_{n+1} \omega-S_{n} \omega\right\|= & \sup _{\omega \in B}\left\|\left(1-\gamma_{n+1}\right) \omega+\gamma_{n+1} T_{n+1} \omega-\left(\left(1-\gamma_{n}\right) \omega+\gamma_{n} T_{n} \omega\right)\right\| \\
\leq & \left|\gamma_{n+1}-\gamma_{n}\right| \sup _{\omega \in B}\|\omega\|+\gamma_{n+1} \sup _{\omega \in B}\left\|T_{n+1} \omega-T_{n} \omega\right\| \\
& +\left|\gamma_{n+1}-\gamma_{n}\right| \sup _{\omega \in B}\left\|T_{n} \omega\right\| \\
\leq & \left|\gamma_{n+1}-\gamma_{n}\right| M_{3}+\sup _{\omega \in B}\left\|T_{n+1} \omega-T_{n} \omega\right\|,
\end{aligned}
$$

where $M_{3}=\sup _{n \geq 1}\left\{\|\omega\|,\left\|T_{n} \omega\right\|\right\}$. From the condition $(C 3)$ and $\left\{T_{n}\right\}$ satisfies the AKTT-condition, then we have 


$$
\sum_{n=1}^{\infty} \sup _{\omega \in B}\left\|S_{n+1} \omega-T_{n} \omega\right\|<\infty,
$$

that is $\left\{S_{n}\right\}$ satisfies the AKTT-condition, we can define nonexpansive mapping $S: C \rightarrow$ $C$ by $S x=\lim _{n \rightarrow \infty} S_{n} x$ for all $x \in C$. Since $\left\{\gamma_{n}\right\}$ is bounded, there exits a subsequence $\left\{\gamma_{n_{i}}\right\}$ of $\left\{\gamma_{n}\right\}$ such that $\gamma_{n_{i}} \rightarrow v$ as $i \rightarrow \infty$. It follows that

$$
S x=\lim _{i \rightarrow \infty} S_{n_{i}} x=\lim _{i \rightarrow \infty}\left[\left(1-\gamma_{n_{i}}\right) x+\gamma_{n_{i}} T_{n_{j}} x\right]=(1-v) x+v T x, \quad \forall x \in C .
$$

That is, $\operatorname{Fix}(S)=\operatorname{Fix}(T)$. Hence, $\operatorname{Fix}(S)=\bigcap_{n=1}^{\infty} \operatorname{Fix}\left(T_{n}\right)=\bigcap_{n=1}^{\infty} \operatorname{Fix}\left(S_{n}\right)=\Omega$. We observe that

$$
\begin{aligned}
\left\|x_{n}-S_{n} x_{n}\right\| & \leq\left\|x_{n}-x_{n+1}\right\|+\left\|x_{n+1}-S_{n} x_{n}\right\| \\
& =\left\|x_{n}-x_{n+1}\right\|+\left\|Q_{C}\left[\alpha_{n} \gamma V x_{n}+\left(I-\alpha_{n} \mu F\right) S_{n} x_{n}\right]-Q_{C} S_{n} x_{n}\right\| \\
& \leq\left\|x_{n}-x_{n+1}\right\|+\left\|\alpha_{n} \gamma V x_{n}+\left(I-\alpha_{n} \mu F\right) S_{n} x_{n}-S_{n} x_{n}\right\| \\
& =\left\|x_{n}-x_{n+1}\right\|+\alpha_{n}\left\|\gamma V x_{n}-\mu F S_{n} x_{n}\right\| .
\end{aligned}
$$

From the condition (C1) and (3.16), we have

$$
\lim _{n \rightarrow \infty}\left\|x_{n}-S_{n} x_{n}\right\|=0 .
$$

On the other hand, we observe that

$$
\begin{aligned}
\left\|x_{n}-S x_{n}\right\| & \leq\left\|x_{n}-S_{n} x_{n}\right\|+\left\|S_{n} x_{n}-S x_{n}\right\| \\
& \leq\left\|x_{n}-S_{n} x_{n}\right\|+\sup _{\omega \in\left\{x_{n}\right\}}\left\|S_{n} \omega-S \omega\right\|,
\end{aligned}
$$

which implies by Lemma 2.6 and (3.17) that

$$
\lim _{n \rightarrow \infty}\left\|x_{n}-S x_{n}\right\|=0 .
$$

Next, we show that

$$
\lim \sup _{n \rightarrow \infty}\left\langle(\gamma V-\mu F) x^{*}, j_{q}\left(x_{n}-x^{*}\right)\right\rangle \leq 0,
$$

where $x^{*}$ is the same as in Theorem 3.4. To show this, we take a subsequence $\left\{x_{n_{i}}\right\}$ of $\left\{x_{n}\right\}$ such that

$$
\lim \sup _{n \rightarrow \infty}\left\langle(\gamma V-\mu F) x^{*}, j_{q}\left(x_{n}-x^{*}\right)\right\rangle=\lim _{i \rightarrow \infty}\left\langle(\gamma V-\mu F) x^{*}, j_{q}\left(x_{n_{i}}-x^{*}\right)\right\rangle .
$$

By reflexivity of a Banach space $X$ and boundedness of $\left\{x_{n}\right\}$, there exists a subsequence $\left\{x_{n_{i}}\right\}$ of $\left\{x_{n}\right\}$ such that $x_{n_{i}} \rightarrow z$ as $i \rightarrow \infty$. It follows from (3.18) and Lemma 3.2 that $z \in \Omega$. Since Banach space $X$ has a weakly sequentially continuous generalized duality mapping, we obtain that

$$
\begin{aligned}
\lim \sup _{n \rightarrow \infty}\left\langle(\gamma V-\mu F) x^{*}, j_{q}\left(x_{n}-x^{*}\right)\right\rangle & =\lim _{i \rightarrow \infty}\left\langle(\gamma V-\mu F) x^{*}, j_{q}\left(x_{n_{i}}-x^{*}\right)\right\rangle \\
& =\left\langle(\gamma V-\mu F) x^{*}, j_{q}\left(z-x^{*}\right)\right\rangle \leq 0 .
\end{aligned}
$$

Finally, we show that $x_{n} \rightarrow x^{*}$ as $n \rightarrow \infty$. Setting $y_{n}=\alpha_{n} \gamma V x_{n}+\left(I-\alpha_{n} \mu F\right) S_{n} x_{n}, \forall n \geq 1$. Then, we can rewrite (3.12) as $x_{n+1}=Q c y_{n}$. It follows from Lemmas 2.1 and 2.3 that 


$$
\begin{aligned}
\left\|x_{n+1}-x^{*}\right\|^{q}= & \left\langle y_{n}-x^{*}, j_{q}\left(x_{n+1}-x^{*}\right)\right\rangle+\left\langle Q_{C} y_{n}-y_{n}, j_{q}\left(Q_{C} y_{n}-x^{*}\right)\right\rangle \\
\leq & \left\langle y_{n}-x^{*}, j_{q}\left(x_{n+1}-x^{*}\right)\right\rangle \\
= & \alpha_{n}\left\langle\gamma V x_{n}-\mu F x^{*}, j_{q}\left(x_{n+1}-x^{*}\right)\right\rangle+\left\langle\left(I-\alpha_{n} \mu F\right)\left(S_{n} x_{n}-x^{*}\right), j_{q}\left(x_{n+1}-x^{*}\right)\right\rangle \\
= & \alpha_{n}\left\langle\gamma V x_{n}-V x^{*}, j_{q}\left(x_{n+1}-x^{*}\right)\right\rangle+\alpha_{n}\left\langle\gamma V x^{*}-\mu F x^{*}, j_{q}\left(x_{n+1}-x^{*}\right)\right\rangle \\
& +\left\langle\left(I-\alpha_{n} \mu F\right)\left(S_{n} x_{n}-x^{*}\right), j_{q}\left(x_{n+1}-x^{*}\right)\right\rangle \\
\leq & \alpha_{n} \gamma L\left\|x_{n}-x^{*}\right\|\left\|x_{n+1}-x^{*}\right\|^{q-1}+\alpha_{n}\left\langle\gamma V x^{*}-\mu F x^{*}, j_{q}\left(x_{n+1}-x^{*}\right)\right\rangle \\
& +\left(1-\alpha_{n} \tau\right)\left\|x_{n}-x^{*}\right\|\left\|x_{n+1}-x^{*}\right\|^{q-1} \\
= & \left(1-(\tau-\gamma L) \alpha_{n}\right)\left\|x_{n}-x^{*}\right\|\left\|x_{n+1}-x^{*}\right\|^{q-1}+\alpha_{n}\left\langle\gamma L x^{*}-\mu F x^{*}, j_{q}\left(x_{n+1}-x^{*}\right)\right\rangle \\
\leq & \left(1-(\tau-\gamma L) \alpha_{n}\right)\left[\frac{1}{q}\left\|x_{n}-x^{*}\right\|^{q}+\left(\frac{q-1}{q}\right)\left\|x_{n+1}-x^{*}\right\|^{q}\right] \\
& +\alpha_{n}\left\langle\gamma V x^{*}-\mu F x^{*}, j_{q}\left(x_{n+1}-x^{*}\right)\right\rangle,
\end{aligned}
$$

which implies that

$$
\begin{aligned}
\left\|x_{n+1}-x^{*}\right\|^{q} & \leq \frac{1-(\tau-\gamma L) \alpha_{n}}{1+(q-1)(\tau-\gamma L) \alpha_{n}}\left\|x_{n}-x^{*}\right\|^{q}+\frac{q \alpha_{n}}{1+(q-1)(\tau-\gamma L) \alpha_{n}}\left\langle\gamma V x^{*}-\mu F x^{*}, j_{q}\left(x_{n+1}-x^{*}\right)\right\rangle \\
& \leq\left(1-(\tau-\gamma L) \alpha_{n}\right)\left\|x_{n}-x^{*}\right\|^{q}+\frac{q \alpha_{n}}{1+(q-1)(\tau-\gamma L) \alpha_{n}}\left\langle\gamma V x^{*}-\mu F x^{*}, j_{q}\left(x_{n+1}-x^{*}\right)\right\rangle .
\end{aligned}
$$

$\begin{array}{llllll}\text { Put } & a_{n} & = & (\tau & - & \gamma L) \alpha_{n}\end{array}$ $b_{n}=\frac{q}{\left(1+(q-1)(\tau-\gamma L) \alpha_{n}\right)(\tau-\gamma L)}\left\langle\gamma V x^{*}-\mu F x^{*}, j_{q}\left(x_{n+1}-x^{*}\right)\right\rangle . \quad$ Then

reduces to formula

$$
\left\|x_{n+1}-x^{*}\right\|^{q} \leq\left(1-a_{n}\right)\left\|x_{n}-x^{*}\right\|^{q}+a_{n} b_{n} .
$$

It follows from the condition $(C 1)$ and (3.19) that $\sum_{n=1}^{\infty} a_{n}=\infty$ and $\lim \sup _{n \rightarrow \infty} b_{n} \leq$ 0 . From Lemma 2.4, we obtain that $x_{n} \rightarrow x^{*}$ as $n \rightarrow \infty$. This completes the proof.

Remark 3.6. Note that Lemma 3.1 is quite similar to the result of Yamada [19] which is obtained in a real Hilbert space but we extended that result to a real $q$-uniformly smooth Banach space.

Remark 3.7. Theorems 3.4 and 3.8 extend and generalize the main result of Ceng et al. [21] in the following ways:

(i) From a real Hilbert space to a real $q$-uniformly smooth Banach space which admits a weakly sequentially continuous generalized duality mapping.

(ii) From a nonexpansive mapping to a countable family of a strict pseudo-contractions mapping.

From Lemmas 2.7, 2.8 and Theorem 3.8, we obtain the following result.

Theorem 3.8. Let $C$ be a nonempty, closed and convex subset of a real q-uniformly smooth Banach space $X$ which admits a weakly sequentially continuous generalized duality mapping $j_{q}$ from $X$ into $X^{*}$. Let $Q_{c}$ be a sunny nonexpansive retraction such that $Q_{c}$ is an orthogonal from $X$ onto $C$. Let $F: C \rightarrow X$ be a $\kappa$-Lipschitzian and $\eta$ strongly accretive operator with constants $\kappa, \eta>0, V: C \rightarrow X$ be an L-Lipschitzian

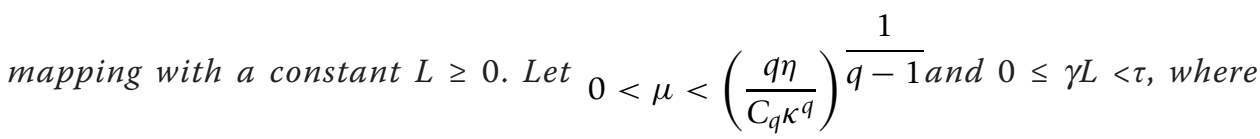
$S_{n} x:=\left(1-\gamma_{n}\right) x+\gamma_{n} \sum_{k=1}^{\infty} \mu_{n}^{k} S_{k} x$. Let $\left\{S_{k}\right\}_{k=1}^{\infty}: C \rightarrow C$ be a sequence of $\lambda_{k^{-s t r i c t}}$ 
pseudo-contractions such that $\bigcap_{k=1}^{\infty} \operatorname{Fix}\left(S_{k}\right) \neq \emptyset$ and $\lambda:=\inf \left\{\lambda_{k}: k \in \mathbb{N}\right\}>0$. Define a mapping $S_{n} x:=\left(1-\gamma_{n}\right) x+\gamma_{n} \sum_{k=1}^{\infty} \mu_{n}^{k} S_{k} x$ for all $x \in C$ and $n \geq 1$. Let $\left\{x_{n}\right\}$ be a sequence defined by $x_{1} \in C$ and

$$
x_{n+1}=Q_{C}\left[\alpha_{n} \gamma V x_{n}+\left(I-\alpha_{n} \mu F\right) S_{n} x_{n}\right], \quad \forall n \geq 1,
$$

where $\left\{\alpha_{n}\right\}$ and $\left\{\gamma_{n}\right\}$ are sequences in $(0,1)$ which satisfy the conditions $(C 1)-(C 3)$ of Theorem 3.8 and $\left\{\mu_{n}^{k}\right\}$ is a sequence which satisfies the conditions (i)-(iii) of Lemma 2.8. Let $T: C \rightarrow C$ be the mapping defined by $T x=\sum_{k=1}^{\infty} \mu^{k} S_{k} x$ for all $x \in C$. Then the sequence $\left\{x_{n}\right\}$ defined by (3.21) converges strongly to $x^{*} \in \bigcap_{k=1}^{\infty} \operatorname{Fix}\left(S_{k}\right)$ as $n \rightarrow \infty$, which $x *$ is the unique solution of the variational inequality

$$
\left\langle(\mu F-\gamma V) x^{*}, j_{q}\left(x^{*}-z\right)\right\rangle \leq 0, \quad \forall z \in \bigcap_{k=1}^{\infty} \operatorname{Fix}\left(S_{k}\right) .
$$

\section{Acknowledgements}

This research was supported by the Centre of Excellence in Mathematics under the Commission on Higher Education, Thailand (under Grant No. RG-1-54-02-1). The first author was partially supported by the Centre of Excellence in Mathematics, the Commission on High Education, Thailand for the Ph.D. Program at King Mongkut's University of Technology Thonburi (KMUTT).

\section{Author details}

${ }^{1}$ Department of Mathematics, Faculty of Science, King Mongkut's University of Technology Thonburi (KMUTT) Bangmod, Bangkok 10140, Thailand ${ }^{2}$ Centre of Excellence in Mathematics, CHE, Si Ayutthaya Rd., Bangkok 10400, Thailand

\section{Authors' contributions}

All authors contributed equally and significantly in writing this article. All authors read and approved the final manuscript.

\section{Competing interests}

The authors declare that they have no competing interests.

Received: 8 November 2011 Accepted: 23 April 2012 Published: 23 April 2012

\section{References}

1. Takahashi, W: Nonlinear Functional Analysis. Yokohama Publishers, Yokohama (2000)

2. Browder, FE: Petryshyn, Construction of fixed points of nonlinear mappings in Hilbert space. J Math Anal Appl. 20 , 197-228 (1967). doi:10.1016/0022-247X(67)90085-6

3. Chen, $\mathrm{R}$, Song, $Y$, Zhou, $\mathrm{H}$ : Convergence theorems for implicit iteration process for a finite family of continuous pseudocontractive mappings. J. Math. Anal. Appl. 314, 701-709 (2006). doi:10.1016/j.jmaa.2005.04.018

4. Kinderlehrer, D, Stampacchia, G: An Introduction to Variational Inequalities and their Applications. Academic press Inc., New York (1980)

5. Noor, MA: General variational inequalities and nonexpansive mappings. J. Math. Anal. Appl. 331, 810-822 (2007). doi:10.1016/j.jmaa.2006.09.039

6. Yao, Y, Shahzad, N: New methods with perturbations for non-expansive mappings in Hilbert spaces, Fixed Point Theory and Applications. 2011, 79 (2011)

7. Yao, Y, Shahzad, N: Strong convergence of a proximal point algorithm with general errors. Optim Lett

8. Yao, Y, Liou, YC, Chen, CP: Algorithms construction for nonexpansive mappings and inverse-strongly monotone mappings. Taiwanese J. Math. 15, 1979-1998 (2011)

9. Yao, Y, Chen, R, Liou, YC: A unified implicit algorithm for solving the triple-hierarchical constrained optimization problem. Math. Comput. Model. 55, 1506-1515 (2012). doi:10.1016/j.mcm.2011.10.041

10. Yao, Y, Liou, YC, Kang, SM, Yu, Y: Algorithms with strong convergence for a system of nonlinear variational inequalities in Banach spaces. Nonlinear Anal. 74, 6024-6034 (2011). doi:10.1016/j.na.2011.05.079

11. Yao, Y, Liou, YC, Kang, SM: Two-step projection methods for a system of variational inequality problems in Banach spaces. J. Global Optim

12. Bynum, WL: Normal structure coefficients for Banach spaces. Pac. J. Math. 86, 427-436 (2001)

13. Byrne, C: A unified treatment of some iterative algorithms in signal processing and image reconstruction. Inverse Probl. 20, 103-120 (2004). doi:10.1088/0266-5611/20/1/006

14. Podilchuk, Cl, Mammone, RJ: Image recovery by convex projections using a least-squares constraint. J. Optim. Soc. Am. A. 7, 517-521 (1990). doi:10.1364/JOSAA.7.000517 
15. Sezan, MI, Stark, H: Applications of convex projection theory to image recovery in tomography and related areas. In: Stark H (ed.) Image Recovery Theory and Applications. pp. 415-462. Academic Press, Orlando (1987)

16. Youla, D: Mathematical theory of image restoration by the method of convex projections. In: Stark H (ed.) Image Recovery Theory and Applications. pp. 29-77. Academic Press, Orlando (1987)

17. Youla, D: On deterministic convergence of iterations of relaxed projection operators. J. Vis. Commun. Image Represent. 1, 12-20 (1990). doi:10.1016/1047-3203(90)90013-L

18. Marino, G, Xu, HK: A general iterative method for nonexpansive mappings in Hilbert spaces. J. Math. Anal. Appl. 318, 43-52 (2006). doi:10.1016/j.jmaa.2005.05.028

19. Yamada, I: The hybrid steepest descent method for the variational inequality problems over the intersection of fixed point sets of nonexpansive mappings. In: Butnariu D, Censor Y, Reich S (eds.) Inherently Parallel Algorithms in Feasibility and Optimization and Their applications, vol. 8, pp. 473-504. North-Holland, Amsterdam (2001). (Stud. Comput. Math.)

20. Tian, M: A general iterative algorithm for nonexpansive mappings in Hilbert spaces. Nonlinear Anal. Theory Methods Appl. 73, 689-694 (2010). doi:10.1016/.jna.2010.03.058

21. Ceng, LC, Ansari, QH, Yao, JC: Some iterative methods for finding fixed points and for solving constrained convex minimization problems. Nonlinear Anal. Theory Methods Appl. 74, 5286-5302 (2011). doi:10.1016/j.na.2011.05.005

22. James, RC: Orthogonality and linear functionals in normed linear spaces. Trans. Am. Math. Soc. 61, 265-292 (1947). doi:10.1090/S0002-9947-1947-0021241-4

23. Bruck, RE: Nonexpansive projections on subsets of Banach spaces. Pac. J. Math. 47, 341-355 (1973)

24. Xu, HK: Inequalities in Banach spaces with applications. Nonlinear Anal. Theory Methods Appl. 16, 1127-1138 (1991). doi:10.1016/0362-546X(91)90200-K

25. Mitrinović, DS: Analytic Inequalities. Springer-Verlag, New York (1970)

26. Aoyama, K, Kimura, Y, Takahashi, W, Toyoda, M: Approximation of common fixed points of a countable family of nonexpansive mapping in a Banach space. Nonlinear Anal. Theory Methods Appl. 67, 2350-2360 (2007). doi:10.1016/j. na.2006.08.032

27. Boonchari, D, Saejung, S: Weak and strong convergence theorems of an implicit iteration for a countable family of continuous pseudocontractive mappings. J. Comput. Appl. Math. 233, 1108-1116 (2009). doi:10.1016/j.cam.2009.09.007

28. Boonchari, D, Saejung, S: Construction of common fixed points of a countable family of $\lambda$-demicontractive mappings in arbitrary Banach spaces. Appl. Math. Comput. 216, 173-178 (2010). doi:10.1016/j.amc.2010.01.027

doi:10.1186/1687-1812-2012-65

Cite this article as: Sunthrayuth and Kumam: Iterative methods for variational inequality problems and fixed point problems of a countable family of strict pseudo-contractions in a q-uniformly smooth Banach space. Fixed Point Theory and Applications 2012 2012:65.

\section{Submit your manuscript to a SpringerOpen ${ }^{\odot}$ journal and benefit from:}

Convenient online submission

- Rigorous peer review

- Immediate publication on acceptance

- Open access: articles freely available online

- High visibility within the field

- Retaining the copyright to your article

Submit your next manuscript at $\boldsymbol{s p r i n g e r o p e n . c o m ~}$ 